\title{
Small-RNA sequencing libraries with greatly reduced adaptor-dimer background
}

Conventional methods for preparing small-RNA-seq libraries by adaptor ligation generate a significant amount of adaptor dimer, thereby resulting in wasted sequencing reads. These methods also do not capture small 5'-capped and 5'-triphosphorylated RNAs. The ScriptMiner ${ }^{\text {TM }}$ small-RNA-seq library preparation technology overcomes these limitations. ScriptMiner ${ }^{\mathrm{Tm}}$ small-RNA libraries contain greatly reduced amounts of adaptor-dimer when compared to conventional methods and also generate coverage that is characteristic of the entire small-RNA transcriptome.

The small-RNA transcriptome contains a diverse array of RNAs, such as microRNA (miRNA), small nucleolar RNA (snoRNA), small nuclear RNA (snRNA), small interfering RNA (siRNA) and piwi-interacting RNA (piRNA). The biogenesis of these classes of RNAs results in a variety of different modifications at the $5^{\prime}$ end: $5^{\prime}$-monophosphate $\left(5^{\prime} \mathrm{pN}\right)$, $5^{\prime}$-triphosphate (5'pppN) or 5'-cap (GpppN). In addition, the $3^{\prime}$ end of the RNA may be modified to include a $2^{\prime}-\mathrm{O}$-methyl, $3^{\prime}-\mathrm{OH}$ instead of the normal 2', $3^{\prime}-\mathrm{OH}$.

Current methods of preparing small-RNA-seq libraries involve ligation-tagging (adaptor-ligation) of the $3^{\prime}$ and 5' ends of the RNA, reverse transcription of the di-tagged RNA into CDNA, and PCR amplification. However, these methods suffer from two major drawbacks. First, they amplify a significant amount of adaptor-dimer that reduces the efficiency of the $5^{\prime}$ ligation reaction and contaminates the sequencing library, leading to a large number of nonproductive sequencing reads. Second, the conventional methods do not capture small 5'-capped and 5'-triphosphorylated RNAs.

The ScriptMiner $^{\text {TM }}$ small-RNA-seq library preparation method significantly reduces the amount of adaptor-dimers in the library and enables the user to capture the entire small-RNA transcriptome, including small 5'-capped and 5'-triphosphorylated RNAs. The result is a more sensitive and comprehensive representation of the small transcriptome in the library.

\section{Overview}

Figure 1 presents an overview of the ScriptMiner ${ }^{\mathrm{TM}}$ process. Briefly, total RNA or size-selected RNA is tagged at its $3^{\prime}$ end with a preadenylated $3^{\prime}$-adaptor oligonucleotide. A significant proportion of the excess

\section{Jim Pease}

EPICENTRE Biotechnologies, Madison, Wisconsin, USA

Correspondence should be addressed to: Jim Pease. E-mail: jim.pease@epibio.com. 3'-adaptor oligonucleotide, which can form undesired adaptor-dimers, is then enzymatically removed. The default procedure provides the option to tag only 5' monophosphorylated RNAs, such as miRNA. An alternative step-treatment with tobacco acid pyrophosphatase

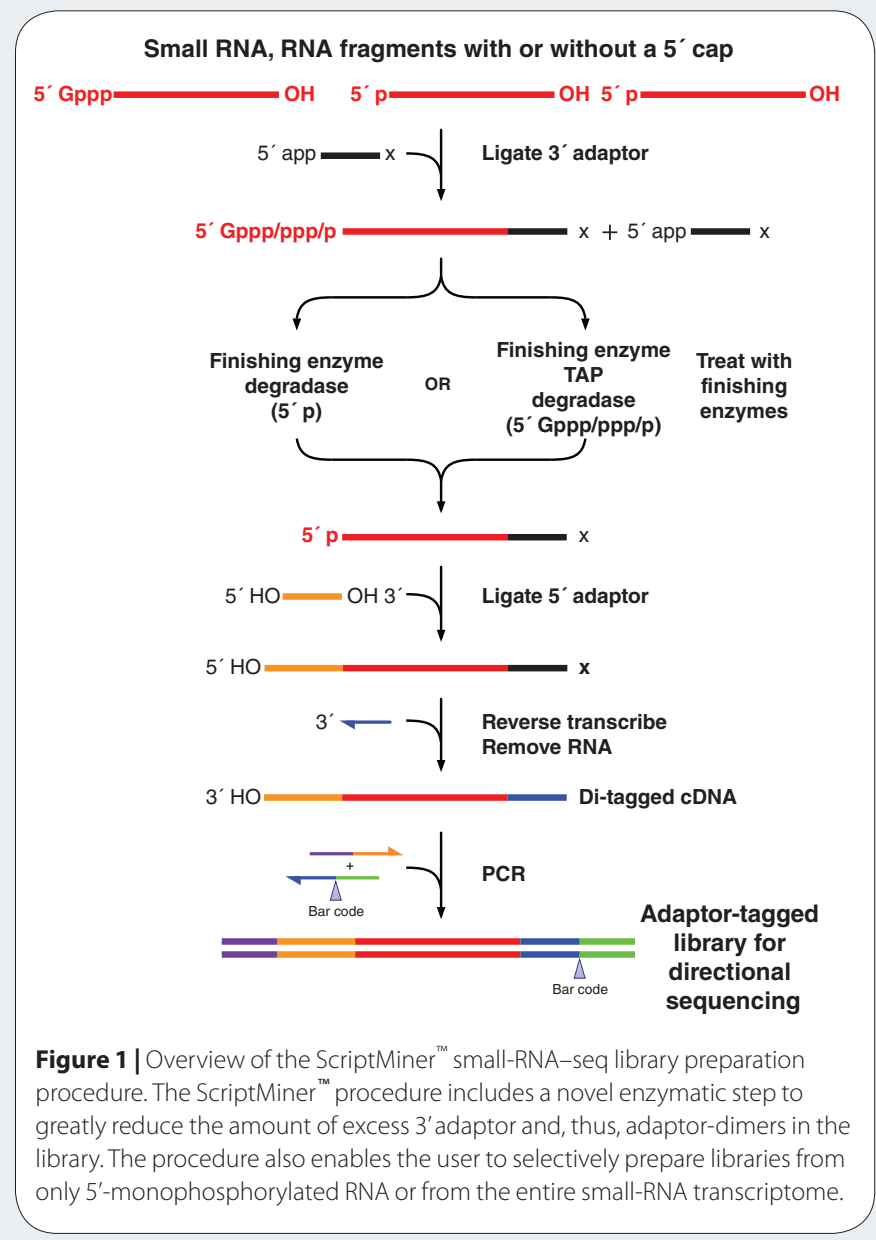


Table 1 | Summary of sequencing data from ScriptMiner libraries with and without TAP treatment. The ScriptMiner library was prepared including TAP treatment to permit capture of the entire small-RNA transcriptome. The untreated ('No-TAP') library captured only 5'-monophosphorylated small RNA. BrRR, human brain reference RNA; HeLa, HeLa total RNA.

\begin{tabular}{ccccc}
\hline RNA sample & Enzyme treatment & Reads passing filter & Reads aligned to hg19 & \% reads aligned \\
\hline BrRR & TAP & $1,384,314$ & $1,380,799$ & 99.75 \\
\hline BrRR & No TAP & 712,841 & 711,926 & 99.87 \\
\hline HeLa & TAP & $1,560,105$ & $1,555,406$ & 99.70 \\
\hline HeLa & No TAP & 662,365 & 661,362 & 99.85 \\
\hline
\end{tabular}

(TAP: supplied in the kits) — enables capture of the entire small-RNA transcriptome. The di-tagged RNA is reverse-transcribed into cDNA, and the cDNA amplified by PCR. In addition to amplifying the library, the PCR also incorporates the necessary platform-specific adaptor sequences into the library and adds a barcode (index read) to the library, if desired. The PCR-amplified library is gel-purified, and the extracted small-RNA library is ready for cluster generation before sequencing.

\section{Reduced adaptor-dimer}

The ScriptMiner ${ }^{\text {TM }}$ procedure uses an optimized strategy for degrading excess 3 ' adaptor oligonucleotide in order to suppress the formation of undesired adaptor-dimers. Figure 2 shows the greatly reduced level

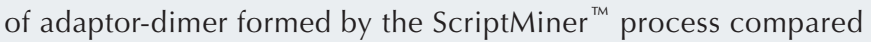
to a conventional small-RNA-seq library method. By significantly reducing the amount of adaptor-dimer, more of the desired smallRNA transcriptome is amplified by PCR, and less adaptor-dimer contaminates the final sequencing library.

\section{The ScriptMiner ${ }^{\mathrm{TM}}$ method captures the entire small-RNA} transcriptome

Following degradation of excess 3' adaptor oligonucleotide, the user has the option of preparing libraries either from small RNA with a 5'-monophosphate (such as miRNA) or from the entire smallRNA transcriptome, depending on the enzymes chosen in the degradation steps (Fig. 1). By treating the sample with TAP, small RNA with a 5' monophosphate, a 5' triphosphate or a 5' cap will all be included in the library. Table 1 shows libraries prepared from only 5'-monophosphorylated RNA ('No TAP') and from TAP-treated samples. The TAP-treated library produced twice as many aligned reads as the 'No-TAP' library, indicating that the TAP-treated library captured more of the small-RNA transcriptome than conventionally produced ('No-TAP') libraries.

\section{Conclusions}

The ScriptMiner ${ }^{\mathrm{TM}}$ procedure significantly reduces the amount of adaptor-dimers in small-RNA libraries compared to conventional methods, thereby reducing the number of wasted sequencing reads. ScriptMiner $^{\text {TM }}$ libraries can be prepared from the whole small-RNA transcriptome, providing a more detailed picture of the regulatory processes that are mediated by small RNAs within a cell. Current

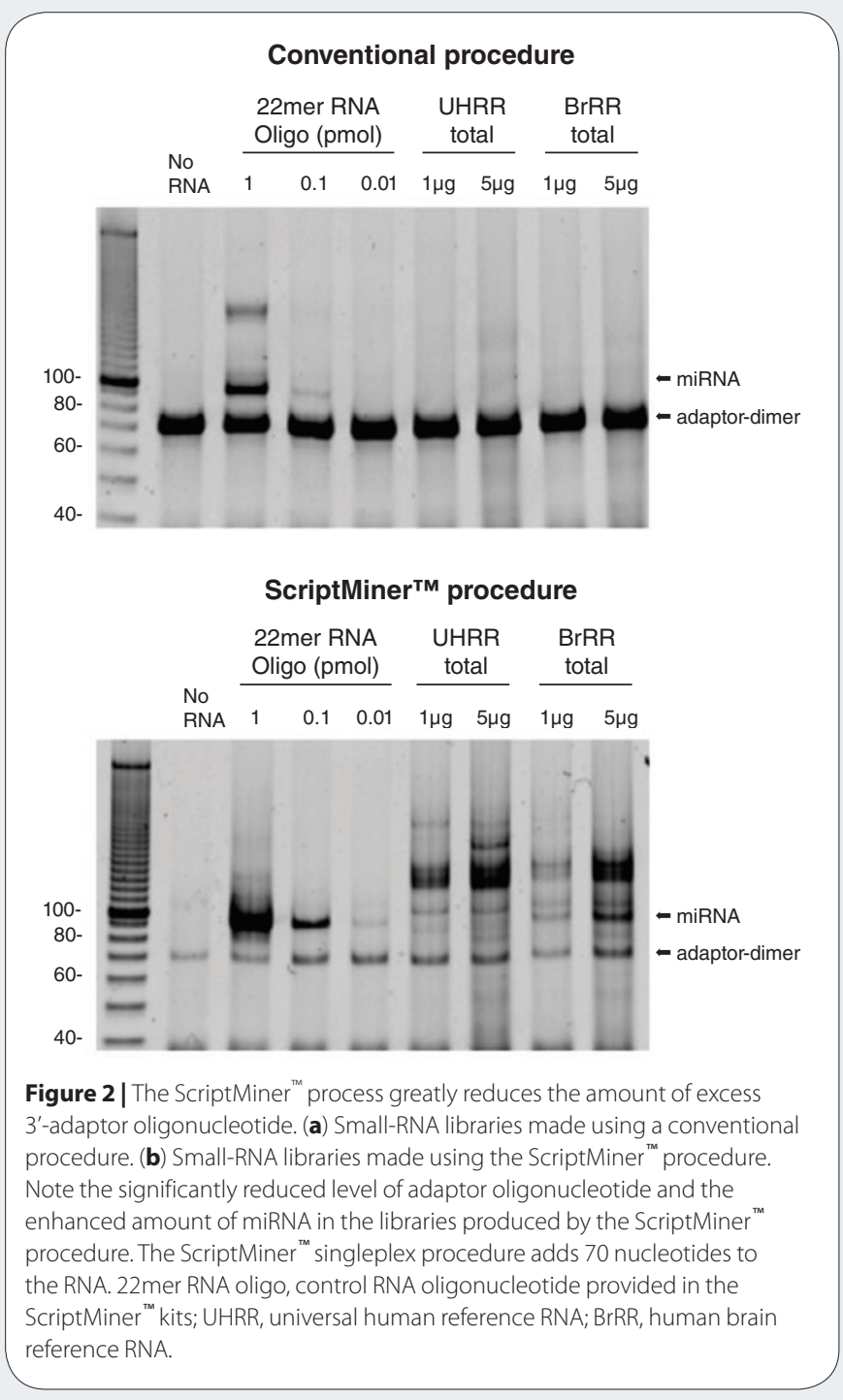

ScriptMiner ${ }^{T M}$ kits permit the preparation of both non-barcoded (singleplex) and barcoded (multiplex) Illumina ${ }^{\circledR}$-compatible libraries.

\section{ACKNOWLEDGMENTS}

We are grateful to Scott Kuersten and Ramesh Vaidyanathan for the data used in this application note.

This article was submitted to Nature Methods by a commercial organization and has not been peer reviewed. Nature Methods takes no responsibility for the accuracy or otherwise of the information provided. 